ermöglichte auch eine annähernde Dichtebestimmung von geschmolzenem $\mathrm{UO}_{2} \mathrm{Cl}_{2}$, indem das von der Schmelze eingenommene Volumen nach dem Herauslösen des erstarrten $\mathrm{UO}_{2} \mathrm{Cl}_{2}$ mit $\mathrm{H}_{2} \mathrm{O}$ durch Auswägen mit Wasser bestimmt wurde. Unter Berücksichtigung der bei dieser etwas primitiven Methode möglichen Fehlerquellen dürfte die Dichte von $\mathrm{UO}_{2} \mathrm{Cl}_{2}$ in einem
Temperaturbereich von $580^{\circ}$ bis $680^{\circ} \mathrm{C}$ zu $4,0 \pm 0,1$ angenommen werden können.

Die Arbeit wurde von Herrn Dr. A. K le m m angeregt, dem wir auch für Ratschläge bei der Durchführung danken. Genauere Angaben über die im Verlauf der Untersuchungen durchgeführten Messungen, Analysen usw. sowie über die benutzte Literatur sind aus der Diplomarbeit von L. O chs ersichtlich.

\title{
Zur Photochemie dicker Schichten I
}

\author{
Von Isolde Hausser $(\dagger)$, Karl H. Hausser und Udo Wegner \\ Aus dem Max-Planck-Institut für medizinische Forschung, Heidelberg, \\ Abteilung für physikalische Therapie \\ (Z. Naturforschg. 7 b, 639-644 [1952]; eingegangen am 22. März 1952)
}

\begin{abstract}
Die mit Hilfe der Poisson-Statistik berechneten theoretischen Umwandlungskurven sind nur für photochemische Vorgänge an Lösungen sehr kleiner Konzentration und Schichtdicke gültig. Da diese Voraussetzung im Experiment nicht immer realisierbar ist, werden in dem hier vorliegenden I. Teil die Umwandlungskurven für photochemische Prozesse in Lösungen beliebiger Konzentration und Schichtdicke unter Vernachlässigung der Diffusion berechnet und diesen dann in einem folgenden II. Teil die entsprechenden Kurven unter Berücksichtigung der Diffusion gegenübergestellt. Es wird diskutiert, inwieweit auch bei optisch dickeren Schichten aus der Form der Umwandlungskurven eindeutige Schlüsse auf die Art des Vorganges gezogen werden können und welche Folgerungen sich auf die Analyse der DosisEffektkurven bei biologischen Objekten ergeben.
\end{abstract}

$\mathrm{I}$ $\mathrm{n}$ einer früheren Arbeit von einem von uns ${ }^{1}$ wurde die Quantenbilanz photochemischer Prozesse (an organischen Molekülen) untersucht. Dabei wurden zur Auswertung der Meßergebnisse Methoden der mathematischen Statistik verwandt, wie sie in der biophysikalischen Strahlenforschung unter dem Namen "Treffertheorie“ allgemein benutzt werden. Diese Untersuchungen wurden an Lösungen kleiner Konzentration und geringer Schichtdicke durchgeführt, in denen die Absorption der eingestrahlten Lichtintensität so gering ist, daß man die Wahrscheinlichkeit von einem Lichtquant getroffen zu werden in erster Näherung für alle Moleküle als gleich groß annehmen kann. Solche Lösungen sollen in Zukunft als "quasimonomolekular" bezeichnet werden, weil man mit ihnen so rechnen kann, als ob sich alle in der Küvette befindlichen Moleküle in einer monomolekularen Schicht senkrecht zum eingestrahlten Licht nebeneinander befänden. Die theoretischen Überlegungen und Rechnungen werden durch diese Voraussetzung sehr wesentlich vereinfacht. Da es jedoch nicht immer möglich sein wird, die experimentellen Verhältnisse an die Forderung der "quasimonomole1 K. H. H a u s ser, Z. Naturforschg. 5 a, 41 [1950]. kularen Schicht" anzupassen, wurde bereits damals die Absicht angekündigt, den Ablauf photochemischer Prozesse in Lösungen beliebiger Konzentration und Schichtdicke theoretisch zu untersuchen. Die Fragestellung dabei lautet: Kann man auch im allgemeinen Fall beliebiger Konzentration und Schichtdicke aus der Form der Umwandlungskurve einen eindeutigen Schluß auf die Art des Vorganges - Ein-, Zwei- oder Mehrtreffervorgang - ziehen, bzw. wie unterscheiden sich die Umwandlungskurven überhaupt von den bei quasimonomolekularer Schicht vorliegenden Kurven? Der Beantwortung dieser Fragen soll die vorliegende Arbeit dienen.

In dem hier untersuchten allgemeinen Fall ist eine Voraussetzung von besonderer Bedeutung, die bei der "quasimonomolekularen Schicht" vernachlässigt werden kann. Es tritt nämlich eine laufende Durchmischung der Moleküle in der Lösung durch Diffusion ein, wobei die Diffusionsgeschwindigkeit von der Art und Konzentration der gelösten Moleküle, von dem Lösungsmittel und von der Temperatur abhängig und in jedem einzelnen experimentellen Fall verschieden sein wird. In der vorliegenden Untersuchung wird zunächst der Ablauf des photochemi- 
schen Vorgangs unter der Voraussetzung der Diffusionsgeschwindigkeit Null, also der völligen Unbeweglichkeit der Moleküle in'der bestrahlten Küvette behandelt. In einem folgenden Teil soll dann die allgemeine Gleichung für den Ablauf der photochemischen Umwandlung unter Einführung einer Diffusionsgeschwindigkeit gegeben werden.

\section{Eintreffervorgänge}

Die übrigen Voraussetzungen für die folgenden Untersuchungen entsprechen den in der früheren Arbeit gegebenen; sie seien hier noch einmal kurz zusammengestellt:

In einer Küvette der Dicke $d$ und der Fläche $F$ senkrecht zum Lichtstrom befinden sich bei Beginn des Versuches $Z_{0}$ Moleküle im Zustand $\alpha, Z_{a}=Z_{0}$. In die Küvette wird ein monochromatischer Lichtstrom der Wellenlänge $\lambda$ mit zeitlich konstanter und über den ganzen Querschnitt gleichmäßig verteilter Intensität $I_{0}$ eingestrahlt, unter dessen Einwirkung sich die Moleküle aus dem Zustand $\alpha$ in den Endzustand $\beta$ irreversibel umwandeln. Der für die $A b-$ sorption eines Quants der Wellenlänge $\lambda$ wirksame Querschnitt eines Moleküls im $\alpha$ - bzw. $\beta$-Zustand sei wieder $f_{a(\lambda)}$ bzw. $f_{\beta(\lambda)}$.

Wir können annehmen, daß in der Küvette die Moleküle innerhalb jeder parallel zu den planen $\mathrm{Be}^{-}$ grenzungsflächen gelegenen dünnen Schicht gleichmäßig verteilt sind. Infolgedessen werden innere, den Vorgang bestimmende Funktionen nur von einer Raum-Koordinate $x$, die die Lage der Schicht parallel zur Begrenzungsfläche bestimmt, und natürlich auch von der Zeit $t$ abhängen. Die zur Zeit $t$ an der Stelle $x$ der Küvette durchgelassene Intensität bezeichnen wir mit $I(x, t)$, so daß $I(0, t)=I_{0}$ wird, da $x=0$ die Eintrittsfläche für die Lichtquanten bedeutet. Bezeichnet man mit $F_{s}(x, t)$ denjenigen Teil der Querschnittsfläche der Küvette an der Schichttiefenstelle $x$ zur Zeit $t$, der für die Lichtquanten der Wellenlänge $\lambda$ undurchlässig ist, so ist offenbar

$$
I(x+\Delta x, t)=I(x, t)\left(1-\frac{F_{s}(x, t) \Delta x}{F}\right)
$$

(von der kleinen zeitlichen Änderung wird dabei abgesehen). Ist nun $q_{a}(x, t)$ die Anzahl der pro Zeiteinheit im Mittel pro Molekül absorbierten Quanten zur Zeit $t$ an der Schichttiefenstelle $x$, so ergibt sich offenbar der Zusammenhang zwischen der Intensität $I$ und dem wirksamen Querschnitt $f_{a}$ eines Moleküls im $a$-Zustand durch

$$
q_{a}(x, t)=\frac{f_{a}}{F} I(x, t) .
$$

Für die für den Lichtquantenstrom undurchlässige Fläche besteht dann gemäß der Definition der wirksamen Querschnittsflächen die Relation

$$
F_{s}(x, t) \Delta x=\left[\left(f_{a} Z_{a}(x, t)+f_{\beta} Z_{\beta}(x, t)\right]\right\lrcorner x,
$$

falls $Z_{a}(x, t) \Delta x$ die Anzahl der Moleküle im $\alpha$-Zustand zur Zeit $t$ innerhalb einer Querschnittsschicht $\Delta x$ in der Tiefe $x$ bezeichnet, und $Z_{\beta}(x, t) \Delta x$ analog definiert ist für die Moleküle im $\beta$-Zustand.

Aus (1) und (3) ergibt sich

$$
\frac{\partial I(x, t)}{\partial x}=-\frac{I(x, t)}{F}\left[f_{a} Z_{a}(x, t)+f_{\beta} Z_{\beta}(x, t)\right] .
$$

Die Kontinuitätsgleichung lautet bei der Annahme völliger Stationarität der Moleküle

$$
Z_{a}(x, t) \Delta x+Z_{\beta}(x, t) \Delta x=Z_{0} \Delta x,
$$

so daß (4) bei Elimination von $Z_{\beta}(x, t)$ übergeht in:

$$
\frac{\partial I(x, t)}{\partial x}=-\frac{I(x, t)}{F}\left[\left(f_{a}-f_{\beta}\right) Z_{a}(x, t)+f_{\beta} Z_{0}\right] .
$$

Auf Grund der Poisson-Statistik errechnet sich die Divergenz $\operatorname{der} Z_{a}(x, t)$ zu:

$$
\frac{\partial Z_{a}}{\partial t}=-Z_{a}(x, t) q_{a}(x, t) .
$$

Verwenden wir die Relation (2) zur Elimination von $q_{a}(x, t)$, so erhalten wir aus $(6)$

$$
Z_{a}(x, t)=Z_{0} e^{-\frac{f_{a}}{F} \int_{0}^{t} I(x, s) \mathrm{d} s} .
$$

Eine 2. Beziehung zwischen $Z_{\alpha}(x, t)$ und der Intensität in der Schichttiefe $x$ ergibt sich aus (4'). Danach ist

$$
I(x, t)=I_{0} e^{\frac{f_{\beta} Z_{0} x}{F}} e^{-\frac{f_{a}-f_{\beta}}{F} \int_{0}^{x} Z_{a}(r, t) \mathrm{d} r .}
$$

Der Bruchteil der Gesamtzahl der Moleküle, der sich zur Zeit $t$ im $\alpha$-Zustand befindet, ist damit

$$
\frac{\hat{Z}_{a}(t)}{Z_{0}}=\frac{1}{d} \int_{0}^{d} \frac{Z_{a b}(x, t)}{Z_{0}} \mathrm{~d} x .
$$

Eine Elimination von $Z_{a}(x, t)$ liefert für

$$
v(x, t)=\int_{0}^{t} I(x, \tau) \mathrm{d} \tau
$$


die Differentialgleichung:

$$
\begin{aligned}
\frac{\partial v}{\partial x}= & -\frac{f_{\beta} Z_{0}}{F} v(x, t) \\
& -\frac{f_{a}-f_{\beta}}{f_{a}} Z_{0}\left(1-e^{\left.-\frac{f_{a}}{F} v(x, t)\right)}\right.
\end{aligned}
$$

mit $v(0, t)=I_{0} t$.

$v(x, t)$ stellt für alle $x \geq 0$ und $t \geqq 0$ bei $f_{a} \geqq f_{\beta}$ eine monoton abnehmende Funktion nach $x$ dar, die stets $>0$ ist und gegen die $x$-Achse konvex ist. Die beiden Extremfälle $f_{\alpha}=f_{\beta}$ und $f_{\beta}=0$ liefern für die Funktion $\hat{Z}_{\alpha}(t)$ Darstellungen durch elementare Funktionen bzw. Quadraturen. Wir wollen diese Fälle näher diskutieren, da sie die Grenzkurven für alle $Z_{a}(t)$ sind, für die $0 \leqq f_{\beta} \leqq f_{a}$ ist.

1. Fall: $f_{\alpha}=f_{\beta}$

Es sei $e^{\frac{f_{a} z_{0} d}{F}}=\dot{\sigma}$ gesetzt, dann wird

$$
\begin{aligned}
& \frac{\hat{Z}_{\beta}(t)}{Z_{0}}=1-\frac{F}{d f_{a} Z_{0}}\left[\operatorname{Ei}\left(-\frac{f_{a} I_{0} t}{F}\right)\right. \\
& \left.-\operatorname{Ei}\left(-\frac{f_{a} I_{0} t}{F} \sigma\right)\right] \text {. }
\end{aligned}
$$

Für $t \rightarrow \infty$ ergibt sich sofort $\hat{Z}_{\beta}(t) \rightarrow 1$.

Für kleinere Werte von $t$ ist:

$$
\frac{\hat{Z}_{\beta}(t)}{Z_{0}}=1-\frac{F}{d f_{a} Z_{0}}\left[\frac{f_{a} Z_{0} d}{F}+\mathrm{O}(\mathrm{t})\right],
$$

d. h. für $t \rightarrow 0$ ist: $\hat{Z}_{\beta}(t) \rightarrow 0$.

Um ein konkretes Beispiel vor Augen zu haben, wählen wir die Konstanten folgendermaßen:

$$
\frac{Z_{0} d}{F}=6 \cdot 10^{16} ; d=1 ; f_{a}=10^{-16} ; \frac{I_{0} f_{a}}{F}=0,1 .
$$

Damit wird:

$\frac{\hat{\mathrm{Z}}_{\beta}(t)}{\mathrm{Z}_{0}}=1-\frac{1}{6}\left[-\mathrm{Ei}\left(-\frac{t}{10} e^{6}\right)+\mathrm{Ei}\left(-\frac{t}{10}\right)\right]$.

Die Halbwertszeit $t_{\mathrm{H}}$, nach Ablauf derer $\hat{Z}_{a}(t)$ $=Z_{\mathbf{0}} / 2$, ist $t_{\mathrm{H}}=114 \mathrm{sec}$.

$\hat{Z}_{\beta}(t) / Z_{0}$ kann leicht gerechnet werden und gibt mit den oben gewählten Konstanten den Verlauf der Kurve 1 in Abb. 1!

Bei diesen und allen folgenden Abbildungen ist jedoch zu beachten, daß es beim Übergang von "quasimonomolekularen" zu optisch dickeren Schich- ten den Sinn verliert, die im Mittel pro Molekül absorbierte Quantenzahl als unabhängige Veränderliche zu wählen, weil diese Größe sich im Verlauf der Umwandlung in unbekannter, von dem Prozentsatz der Umwandlung abhängiger Weise ändert. Es wird daher an Stelle der absorbierten die pro Molekül eingestrahlte Quantenzahl $\left(I_{0} / Z_{0}\right) t$ als Abszisse aufgetragen.

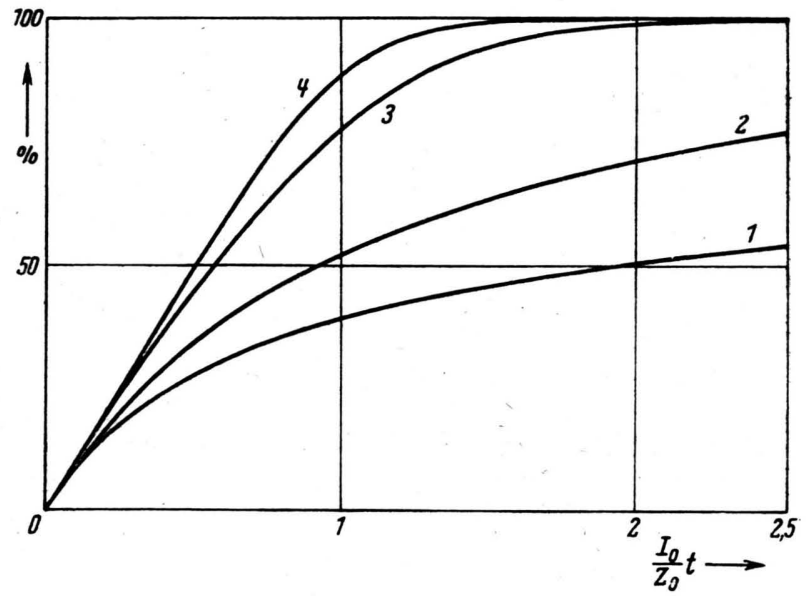

Abb. 1. Berechnete Umwandlungskurve für einen Eintreffervorgang in einer Lösung großer Konzentration und Schichtdicke. Kurven 1-4: Parameter $f_{\beta} / f_{\alpha}=0 ; 0,1$; 0,$5 ; 1$. Abszisse: Anzahl der pro Molekül eingestrahlten Quanten $\left(I_{0} / Z_{0}\right) t$. Ordinate: Umwandlung in Prozent.

2. Fall: $f_{\beta}=0$

Dann ist:

$$
I(x, t)=\frac{I_{0} e^{\frac{f_{a}}{F} I_{0} t} e^{-\frac{f_{a}}{F} \times Z_{0}}}{1-e^{-\frac{f_{a}}{F} Z_{0} x}+e^{\frac{f_{a}}{F}\left(I_{0} t-Z_{0} x\right)}} .
$$

Für $t=0$ ergibt sich:

$$
I(x, 0)=I_{0} e^{-\frac{f_{a} z_{0}}{F} x}
$$

und für $t \rightarrow \infty: I(x, t) \rightarrow I_{0}$, ein physikalisch notwendiges Ergebnis. $I(x, o)=I_{0} e^{-f_{a} z_{0}}{ }^{x}$ stellt aber das Lambert-Beersche Gesetz dar. Weiter ist:

$$
\begin{aligned}
\frac{\hat{Z}_{a}(t)}{Z_{0}}=\frac{F}{f_{a} d Z_{0} \cdot \ln [1} & 1-e^{\frac{f_{a}}{F} I_{0} t} \\
& \left.+e^{\frac{f_{a}}{F}\left(Z_{0} d-I_{0} t\right)}\right] .
\end{aligned}
$$


Die Halbwertszeit $t_{\text {H }}$ ist

$$
t_{\mathrm{H}}=\frac{F}{f_{a} I_{0}} \ln \left(1+e^{\frac{f_{a} z_{0} d}{2 F}}\right)
$$

Mit den obigen Konstanten ergibt sich: $t_{\mathrm{H}}=30,486 \mathrm{sec}$. Die Kurve ist wieder in Abb. 1 als Kurve 4 dargestellt. Weiterhin wurden die Kurven mit $f_{\beta} / f_{a}=1 / 2$; $f_{\beta} / f_{\alpha}=1 / 10$ berechnet.

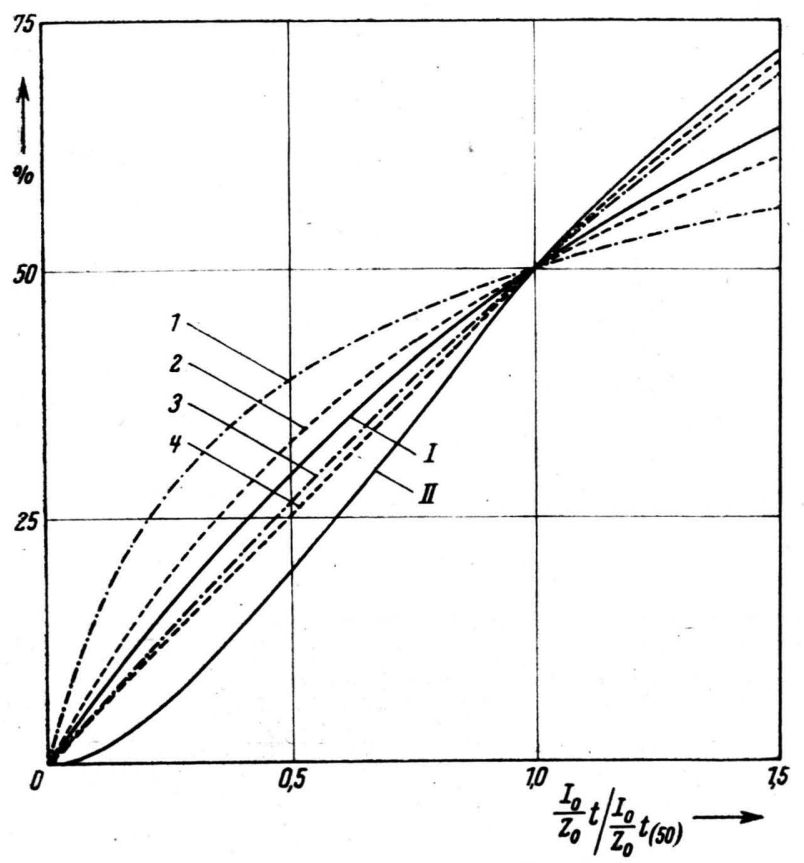

Abb. 2. Kurven von Abb. 1 als Dosis-Effektkurven. Zum Vergleich gewöhnliche Ein- und Zweitrefferkurve (Kurven I und II). Abszisse: Quotient aus der Anzahl der pro Molekül eingestrahlten Quanten $\left(I_{0} / Z_{0}\right) t$ und der für die Umwandlung von 50\% erforderlichen Anzahl der pro Molekül eingestrahlten Quanten $\left(I_{0} / Z_{0}\right) t_{50}$. Ordinate: Umwandlung in Prozent.

Mit den angeführten Konstanten sind die folgenden Gleichungen zu lösen

$$
\frac{\partial w}{\partial x}=-6 \frac{f_{\beta} w}{f_{a}}-\left(1-\frac{f_{\beta}}{f_{a}}\right)\left(1-e^{-6 w}\right),
$$

wobei $w=v(x, t) / Z_{0}$ ist, mit der Anfangsbedingung: $w(0, t)=\left(I_{0} / Z_{0}\right) t=t / 60$.

Für $t$ wurde gewählt:

$t=0,10,20,30,40,50,60,70,80,90,100,120,150$.

$$
\frac{\hat{\mathrm{Z}}_{\beta}(t)}{\mathrm{Z}_{0}}=1-\frac{1}{d} \int_{0}^{d} e^{6 w} \mathrm{~d} x=1-\int_{0}^{1} e^{-6 w} \mathrm{~d} x .
$$

Die Ergebnisse dieser Rechnung sind ebenfalls in Abb. 1 als Kurven 2 bzw. 3 eingezeichnet. In Abb. 2 sind die Kurven 1-4 der Abb. 1 als Dosis-Effektkurven und zum Vergleich die für "quasimonomolekulare Schichten“" gültigen normalen Ein- und Zweitrefferkurven (I und II) aufgetragen. Wie man sieht, bleibt trotz nicht unerheblicher Abweichungen von der Eintrefferkurve bei allen 4 Kurven der Eintreffercharakter deutlich erkennbar; insbesondere münden alle mit steiler Tangente in den Nullpunkt.

\section{Mehrtreffervorgänge}

Im folgenden werden die entsprechenden Gleichungen für einen Zweitreffervorgang abgeleitet. Auf die Behandlung von Vorgängen mit einer Trefferzahl $n \geq 3$ wird verzichtet, weil diese gegenüber dem Zweitreffervorgang nichts grundsätzlich Neues bringen, andererseits der Rechenaufwand mit zunehmenden $n$ so stark ansteigt, daß eine numerische Auswertung sehr zeitraubend wird.

Die am Anfang dieser Arbeit gegebenen Voraussetzungen müssen für Zweitreffervorgänge dahingehend geändert werden, daß ein Molekül durch Absorption eines zweiten Quants im $\beta$-Zustand irreversibel in den Endzustand $\gamma$ umgewandelt wird; die Definitionen für $f_{\gamma}, q_{\gamma}$ usw. sind dieselben wie bei den entsprechenden $\alpha$ - bzw. $\beta$-Größen.

In (2) tritt noch die entsprechende Beziehung hinzu

$$
q_{\beta}=\left(f_{\beta} / F\right) I(x, t) .
$$

Der weitere Verlauf der Rechnung ist dem bei Eintreffervorgängen sehr ähnlich.

Für die für den Lichtquantenstrom undurchlässige Fläche besteht gemäß der Definition der wirksamen Querschnittsfläche die Relation

$$
\begin{aligned}
F_{s}(x, t) \Delta x=\left[f_{a}^{\circ} Z_{a}(x, t)\right. & +f_{\beta} Z_{\beta}(x, t) \\
& \left.+f_{\gamma} Z_{\gamma^{\prime}}(x, t)\right] \Delta x .
\end{aligned}
$$

Das Analogon zu (4) lautet jetzt

$$
\begin{aligned}
\frac{\partial \boldsymbol{I}(\boldsymbol{x}, \boldsymbol{t})}{\partial x}=-\frac{I(x, t)}{F}\left[f_{a} Z_{a}(x, t)\right. & +f_{\beta} Z_{\beta}(x, t) \\
& \left.+f_{\gamma} Z_{\gamma}(x, t)\right] .
\end{aligned}
$$

und das zu $\left(4^{\prime}\right)$ bei Elimination von $Z_{\gamma}(x, t)$ mittels der Kontinuitätsgleichung

$$
\begin{aligned}
\frac{\partial I}{\partial x}=-\frac{I(x, t)}{F} & {\left[\left(f_{a}-f_{, ;}\right) Z_{a}(x, t)\right.} \\
& \left.+\left(f_{\beta}-f_{\gamma}\right) Z_{\beta}(x, t)+f_{\gamma,} Z_{0}\right] .
\end{aligned}
$$


Gl. (6) bleibt bestehen und für die Zahl der Moleküle im $\beta$-Zustand ergibt die Poisson-Statistik:

$$
\begin{aligned}
\frac{\partial Z_{\beta}(x, t)}{\partial t}=- & Z_{\beta}(x, t) q_{\beta}(x, t) \\
& +Z_{a}(x, t) q_{a}(x, t) .
\end{aligned}
$$

Durch (6) und (11) wird $Z_{a}$ und $Z_{\beta}$ vermöge (2) durch $I(x, t)$ ausgedrückt. Substitution dieser Werte in $\left(4^{\prime}\right.$ a) ergibt für

$$
v=\int_{0}^{t} I(x, \tau) d \tau
$$

die 2 Differentialgleichungen $\left(12_{1}\right)$ und $\left(12_{2}\right)$.

1. Fall: $f_{a} \neq f_{\beta}$

$$
\begin{aligned}
& \frac{\partial v}{\partial x}=-\frac{f_{\gamma} Z_{0}}{F} v-Z_{0}\left[\frac{f_{a}-f_{\beta}}{f_{a}}-\frac{f_{\beta}-f_{\gamma}}{f_{a}-f_{\beta}}\right] . \\
& \left(1-e \cdot \frac{f_{a}}{F} v\right)-Z_{0} \frac{f_{a}}{f_{\beta}} \frac{f_{\beta}-f_{\gamma}}{f_{a}-f_{\beta}}\left(1-e^{\left.\frac{f_{\beta}}{F} v\right)}\right.
\end{aligned}
$$

Randbedingung: $v(0, t)=I_{0} t$.

2. Fall: $f_{\alpha}=f_{\beta}$

$$
\begin{aligned}
\frac{\partial v}{\partial x}= & -\frac{f_{\gamma} Z_{0}}{F} v-\frac{2\left(f_{a} f_{\gamma}\right)}{f_{a}} Z_{0}\left(1-e^{-\frac{f_{a}}{F} v}\right) \\
& +\frac{f_{a}-f_{\gamma}}{F} Z_{0} v e^{-\frac{f_{a}}{F} v} .
\end{aligned}
$$

Randbedingung: $v(0, t)=I_{0} t$.

Die Integration dieser Differentialgleichungen geschieht numerisch genau so wie beim Eintreffervorgang. Der Bruchteil der Moleküle, die sich zur Zeit $t$ in der ganzen Küvette im $\gamma$-Zustand befinden, ist damit:

$$
\frac{\hat{Z}_{\gamma}(t)}{Z_{0}}=1-\frac{1}{d} \int_{0}^{d} \frac{Z_{a}(x, t)+Z_{\beta}(x, t)}{Z_{0}} \mathrm{~d} x .
$$

Diese Kurven (im 1. und 2. Falle) beginnen bei $t=0$ mit einer horizontalen Tangente.

Die Kurven steigen konvex zur $t$-Achse gekrümmt an, gehen durch einen Wendepunkt und nähern sich konkav zur $t$-Achse gekrümmt der Asymptote: $\dot{\mathbf{Z}}_{\gamma} / \mathbf{Z}_{\mathbf{0}}=1$.

Beim Mehrtreffervorgang ist die Anzahl der Parameter noch um einen, $f_{\gamma}$, größer als beim Eintreffervorgang. Dadurch ergeben sich sehr viele denkbare Kombinationsmöglichkeiten.
Es soll im folgenden noch der einfachste Fall $f_{\alpha}=f_{\beta}=f_{\gamma}$ betrachtet werden. In diesem Fall ist:

$$
\begin{aligned}
\frac{\hat{Z}_{\gamma}(t)}{Z_{0}}=1-\frac{F}{d f_{a} Z_{0}}\left\{\operatorname{Ei}\left(-\frac{f_{a} I_{0} t}{F}\right)\right. \\
-\operatorname{Ei}\left(-\frac{f_{a} I_{0} t}{F} e^{-\frac{f_{a} Z_{0} d}{F}}\right) \\
\left.+e^{\frac{f_{a} I_{0} t}{F}} e^{-\frac{f_{a} Z_{0} d}{F}}-e^{\frac{f_{a} I_{0} t}{F}}\right\} .
\end{aligned}
$$

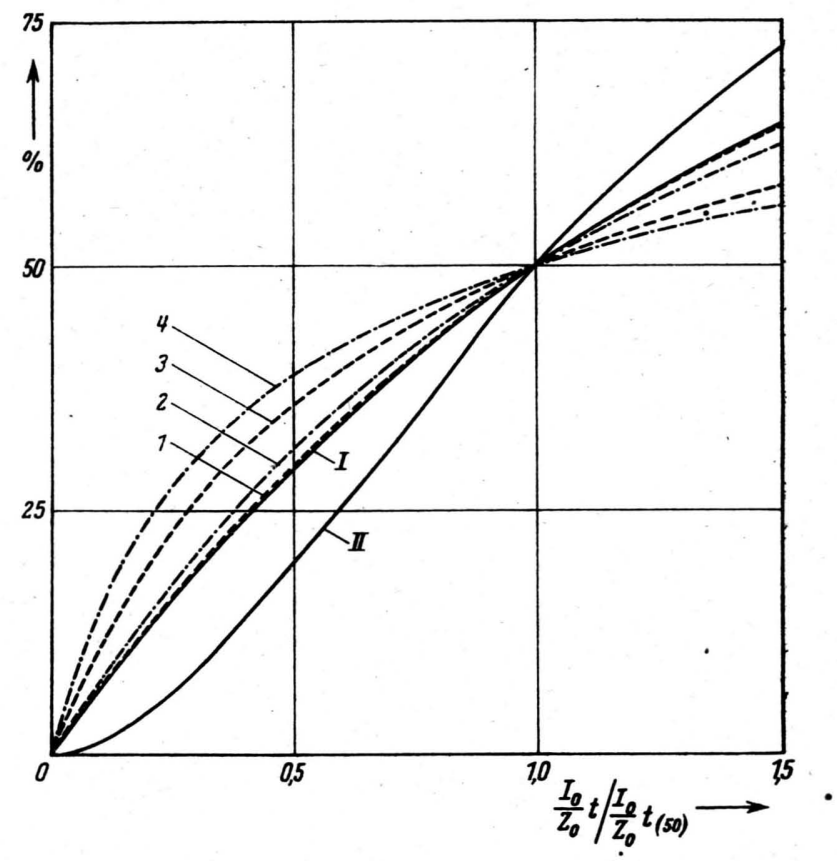

Abb. 3. Berechnete Eintreffer-Dosis-Effektkurven für vier verschiedene optische Schichtdicken. Abszisse: Quotient aus der Anzahl der pro Molekül eingestrahlten Quanten $\left(I_{0} / Z_{0}\right) t$ und der für die Umwandlung von $50 \%$ erforderlichen Anzahl der pro Molekül eingestrahlten Quanten $\left(I_{0} / Z_{0}\right) t_{(50)}$.

In Abb. 3 sind die Eintreffer- und in Abb. 4 die Zweitreffer-Dosis-Effektkurven unter Verwendung derselben Zahlenwerte wie auf S. 641, jedoch für vier verschiedene optische Schichtdicken aufgetragen. Die Lichtabsorption beträgt in beiden Abbildungen bei den Kurven 1-4: 50\%, 90\%, 99\%, 99,75\%. Außerdem sind in beiden Abbildungen die gewöhnlichen, bei „quasimonomolekularer Schicht" gültigen Einund Zweitrefferkurven eingezeichnet. 
Die Abbildungen lassen den großen Einfluß der „optischen Schichtdicke" auf die Form der Um-

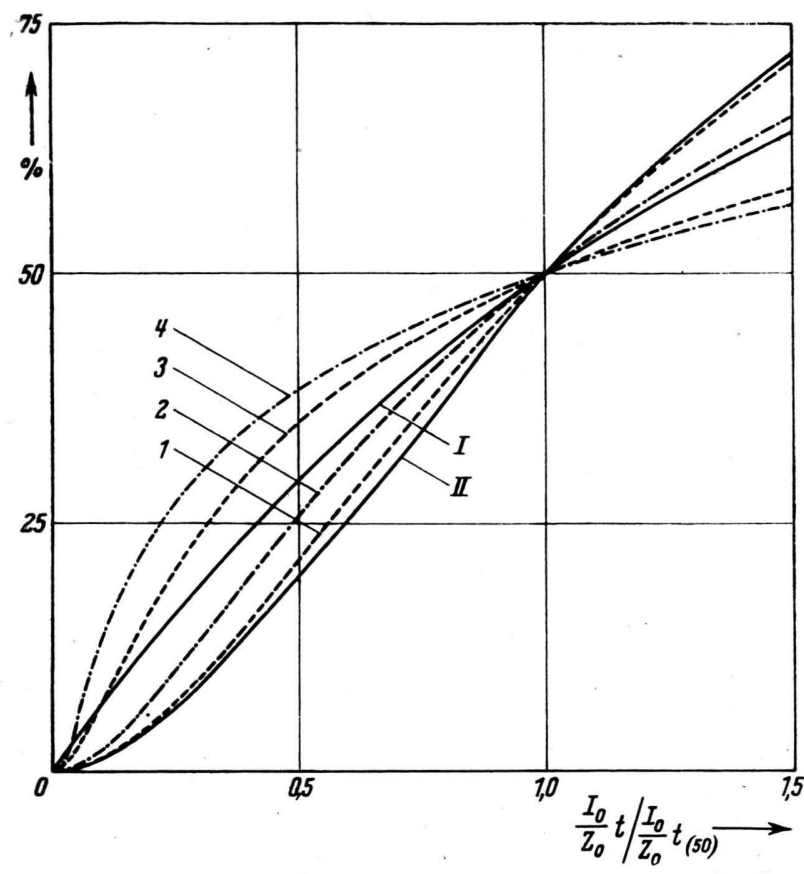

Abb. 4. Berechnete Zweitreffer-Dosis-Effektkurven für vier verschiedene optische Schichtdicken. Abszisse: Quotient aus der Anzahl der pro Molekül eingestrahlten Quanten $\left(I_{0} / Z_{0}\right) t$ und der für die Umwandlung von $50 \%$ erforderlichen Anzahl der pro Molekül eingestrahlten Quanten $\left(I_{0} / Z_{0}\right) t_{(50)}$.

wandlungskurven erkennen. Während sich die für 50\% Absorption berechneten Dosis-Effektkurven nur wenig von den normalen Ein- bzw. Zweitrefferkurven unterscheiden, sind die Abweichungen bei $99 \%$ bereits so groß, daß sogar die Verwechslung einer Zweitrefferkurve mit einer Eintrefferkurve möglich wird (Kurve 3 und 4 in Abb. 4).

Der hier diskutierte Fall, daß sich der Absorptionsquerschnitt für Licht der Umwandlungswellenlänge nicht ändert und die Diffusion während der Umwandlungszeit vernachlässigt werden darf, wird bei photochemischen Prozessen an Lösungen verhältnismäßig selten sein. Bei der Bestrahlung biologischer Objekte mit ultraviolettem oder sichtbarem Licht ${ }^{2}$ dagegen wird dieser Fall der relativ häufigste sein ${ }^{3}$. Man wird daher bei der Analyse solcher Dosis-Effektkurven in jedem Fall prüfen müssen, in welchem Maße die Form der Kurven durch die .,optische Dicke" der bestrahlten Schicht, oder mit anderen Worten, durch die ungleichmäßige Verteilung der Dosis auf das untersuchte Volumen, beeinflußt wird. Dies kann für Ein- und Zweitrefferkurven grob durch Vergleich mit den Abb. 3 und 4 geschehen. Sollen die Abweichungen für einen bestimmten Fall genauer ermittelt werden, so müssen die experimentell gegebenen Zahlenwerte in die Gl. (10) bezw. $\left(12_{1,2}\right)$ eingesetzt und die Dosis-Effektkurven berechnet werden.

2 Bei Bestrahlung mit Röntgen- oder radioaktiver Strahlung sind die Verhältnisse wegen der Sekundärbzw. Tertiär-Elektronen viel komplizierter.

3 Bei biologischen Objekten gelten die berechneten Umwandlungskurven natürlich nur angenähert, da die für eine genaue Gültigkeit notwendige Exaktheit der Voraussetzungen in der Biologie wegen der Inhomogenität des bestrahlten Materials und wegen der biologischen Variabilität nicht gegeben ist. 